\title{
Writing Rounds: An Innovation to Increase Physician Scientific Dissemination
}

Kirsten Winnie, MD | Jeremy T. Jackson, BA | Christy J.W. Ledford, PhD

PRiMER. 2021;5:34.

Published: 9/27/2021 | DOI: 10.22454/PRiMER.2021.178789

\section{Abstract}

Introduction: Many high-quality studies presented at conferences never reach the peer-reviewed literature, most likely because physician authors do not take the next step to fully write up the studies and submit them to a journal. We evaluated a curriculum designed to equip authors with the practical skills to submit research projects to peer-reviewed publication.

Methods: We designed a mixed asynchronous-synchronous longitudinal curriculum, occurring across 4 months via a virtual platform. To evaluate the curriculum, we tracked process and production outcomes and conducted semistructured interviews with participants following participation.

Results: Across two cohorts in 2019, nine participant authors completed the curriculum. Seven participants submitted their studies for publication; two were accepted. In interviews with eight participants, participant authors described the value of the program, expressing intention to participate again and to recommend it to colleagues.

Conclusion: Through a coach-directed writing group, participant authors developed the skills and confidence needed to prepare and submit scientific manuscripts for peer review. Curriculum maintenance and enhancement is ongoing. We plan to scale up this innovation in support of other university departments and medical disciplines, developing an implementation guide to describe needed elements, including technological platforms, qualities of the coach, author recruitment, and group conduct.

\section{Introduction}

While attending regional academic medicine conferences, our practice-based research network (PBRN) team recognized that high-quality studies conducted at network sites were not shared beyond the regional conference. Research shows that our network is not unique. One systematic review summarized that $85 \%$ of unpublished studies were never submitted to a journal, noting this as a failure of authors to write up and submit their work for peer review. ${ }^{1}$ We see this as a critical gap in the scholarly process, since we rely on the clear communication of research findings to continuously improve evidence-based practice.

Previous investigations reveal that unclear professional expectations, lack of confidence, and lack of knowledge about writing for scholarly publication, including not understanding the distinction between content and structure considerations, ${ }^{2}$ are barriers to academic writing for medical faculty and novice researchers. ${ }^{3}$ 
Residents expressed similar concerns in a study of internal medicine residents, in which $79 \%$ of participants reported that learning medical writing was important, but only $3 \%$ reported being thoroughly taught those skills. ${ }^{4}$ One interventional strategy to improve scientific writing and dissemination is academic writing groups, ${ }^{3}$ particularly focused on providing peer support. ${ }^{5,6}$

Encouraged by the success of other virtually-hosted faculty development programs, ${ }^{7}$ we created a virtual writing group program to serve as an educational and supportive resource to physicians across our network.

\section{Methods}

\section{Curriculum Description}

The Writing Rounds curriculum was developed iteratively, using the Six-Step Approach to Curriculum Development. $^{8}$ The curriculum focused on the writing and publication process for first-time authors with the overall goal to facilitate manuscript submission to a journal. Table 1 summarizes the curricular content of Writing Rounds. The mixed asynchronous-synchronous longitudinal curriculum occurred monthly across 4 months via a virtual platform.

Curriculum design was underpinned by self-determination theory (SDT), ${ }^{9}$ which posits that motivation increases when the educational environment meets three needs: autonomy, competence, and relatedness. To encourage autonomy, the curriculum equipped authors to select a target journal and choose a central message for the manuscript. To develop competence, the curriculum included a written curriculum and reference guide that was delivered via group meetings led by a coach, ${ }^{10}$ an individual with medical writing experience (author J.T.J.). To support relatedness needs, the curriculum was designed in the group format to create discussions in virtual meetings monthly with peer authors.

Specific topics for each meeting were selected by the PBRN's research director and publications coordinator in collaboration with a junior faculty member (a member of the target group). In addition to synchronous virtual meetings, authors in the group participated in asynchronous assignments in two forms: assignments for authors to complete on their own manuscript, and a review assignment of the other participants' work. Participants were provided with a workbook reviewing concepts in each topic and assignments to follow each meeting.

\section{Feasibility Testing}

Feasibility testing was conducted in 2018 to determine the optimal sequencing of the curriculum and to select the virtual meeting platform. The coach scheduled four virtual group meetings that included the first author as the participant author, the research director (author C.J.W.L.), and the network clinical research manager. Each week, we tested a different virtual platform for connectivity and group functions as the research director and research manager acted as peers for the physician researcher. Each group member recorded field notes that informed curricular choices about technology use, curricular scope and sequence, and interactivity.

\section{Participant Recruitment}

The PBRN leadership team recruited participant authors who had given poster or podium presentations at the research competition of the Uniformed Services Academy of Family Physicians (USAFP) annual meeting, which is attended by most of our network physicians. Participants in the USAFP research competition had been reviewed and selected by the Academy's Clinical Investigations Committee. We limited invitations to participants who presented original research (in contrast to case reports) and had not previously published a research article in the peer-reviewed literature. The second cohort consisted of participants who were not available for the pilot cohort or who had expressed interest in Writing Rounds after hearing about it from peer 
physicians.

\section{Evaluation Strategy}

The Uniformed Services University of the Health Sciences Institutional Review Board reviewed this multimethod evaluation and (Protocol DBS.2019.029) determined it did not meet the criteria defining research.

First, quantitatively, we tracked participant author attendance, engagement, and production. The coach monitored participant attendance and engagement throughout the rounds, ensuring that participants were completing assignments to meet objectives 1,2, and 3 . After curriculum completion, the coach contacted each participant at 3,6 , and 12 months to assess if authors had fully submitted a manuscript for peer review. We analyzed data using descriptive statistics.

Second, the lead author (K.W.), who had not participated in the groups but is known to the participants, conducted semistructured interviews after the completion of each cohort to collect qualitative feedback. The interviewer recorded detailed field notes from the interviews. We analyzed data through a directed qualitative content analysis approach, ${ }^{11}$ specifically looking for SDT connections.

\section{Results}

Of the 17 physicians invited to participate in Writing Rounds in April 2019, five participated in the spring cohort, and another four participated in the fall cohort. Table 2 presents author characteristics.

Across the sequence of the curriculum, all authors attended and engaged in the first three rounds. At Round four, in each cohort, attendance and engagement declined by one author, which indicated that not all participants accomplished objective three about practicing the skills of peer review. Table 3 presents process and outcome measures by cohort.

Of the nine total participant authors, seven (77.8\%) completed successful submissions of their manuscripts to peer-reviewed publications, meeting objective five of the curriculum.

From those submissions, two manuscripts were accepted at peer-reviewed journals.

Of the nine participants, eight completed interviews about their experience. All participants indicated that they would recommend the program to a colleague and would participate again with a new project. One author shared that Writing Rounds provided "initiative and incentive to move forward."

In regard to autonomy support, authors valued their autonomy in decisions throughout the curriculum. Authors voiced appreciation of the autonomy-supportive environment, which was cultivated by the coach and prepared them to make better decisions. Although authors appreciated autonomy support, they welcomed the "accountability every month."

Authors indicated that their competence increased through the curriculum. Authors described the coach as instrumental in increasing their ability. They also valued the written workbook content, specifically identifying the benefit of seeing the submission process clearly outlined, which made submitting "less scary." Authors' increased competence made them more confident in their peer-reviewing abilities. One author shared, "I would be more critical of conclusions authors draw based on their data [now better understanding that] people may try to stretch their conclusions."

Authors also recognized the role of relatedness in their learning. One author appreciated that "by the end, there is a sense of community and teamwork." Authors specifically valued the multiinstitutional composition of the groups. One author shared that "getting an outside perspective on the research" helped frame findings. The interactivity in the group was essential to building confidence in peer-review skills. One author shared that by 


\section{Discussion}

Through the implementation of a coach-directed writing group, we increased the number of physician authors' submissions to peer review in our PBRN. Participant authors developed the skills and confidence needed to prepare and submit scientific manuscripts for peer review. Quantitative and qualitative evaluation suggests that the curriculum successfully applied the tenets of SDT to increase motivation to submit manuscripts for peer review.

Two innovations facilitated the success of this program: (1) the virtual environment enabled a mix of synchronous and asynchronous group efforts, and (2) the applied skills of an experienced writer as the author coach. Writing Rounds is designed for sustainable, low-resource implementation. The material costs are minimal, as we used teleconferencing software that was free for participants. Universities and networks considering implementing the curriculum will face two potential barriers: access to a suitable coach and willing participants.

We continue curriculum maintenance and enhancement as we plan for additional cohorts of authors. In response to author feedback, we are incorporating more postrounds touchpoints to increase longitudinal support and accountability. As we move forward, we seek feedback from authors about how the program can support author autonomy without feeling controlling. We have also discovered the power of this program to train physicians to be effective peer reviewers and continue to build out this part of the program. Future inquiry will also track how participation in this writing program increases engagement with the PBRN long term.

The evaluation of this curriculum is limited by its design. First, in these pilot cohorts, we use primarily descriptive methods and did not use a comparison group of physicians who did not complete the curriculum. Second, although two of our outcome measures are observational (attendance and engagement), we relied on self-report to measure submission itself.

In the increasingly virtual learning environment we are experiencing in response to COVID-19, ${ }^{12}$ we expect to scale up this innovation in support of other university departments and medical disciplines. In support of this goal, we are developing an implementation guide to facilitate implementation, including technological platforms, qualities of the coach, author recruitment, and group conduct.

\section{Tables and Figures}


Table 1: Curricular Content of Writing Rounds

\begin{tabular}{|c|c|c|c|}
\hline \multicolumn{4}{|c|}{ Program Objectives } \\
\hline \multicolumn{4}{|c|}{ Learners will write an abstract for manuscript submission. } \\
\hline \multicolumn{4}{|c|}{ Learners will identify the journals that best fit the manuscript in terms of topic and audience. } \\
\hline \multicolumn{4}{|c|}{ Learners will understand the journal submission process. } \\
\hline \multicolumn{4}{|c|}{ Learners will successfully complete submission of a research paper. } \\
\hline & $\begin{array}{l}\text { Individual Preparatory } \\
\text { Work (Asynchronous) }\end{array}$ & $\begin{array}{l}\text { Virtual Meeting Discussion Topics } \\
\text { (Synchronous) }\end{array}$ & $\begin{array}{l}\text { Individual Round } \\
\text { Assignment } \\
\text { (Asynchronous) }\end{array}$ \\
\hline $\begin{array}{l}\text { Round } 1 \\
\text { Writing your } \\
\text { abstract }\end{array}$ & Send abstract to coach & $\begin{array}{l}\text { Introductions of authors and projects } \\
\text { How to write a focused abstract }\end{array}$ & $\begin{array}{l}\text { Revise own abstract } \\
\text { Review others' abstracts }\end{array}$ \\
\hline $\begin{array}{l}\text { Round } 3 \\
\text { Peer review } \\
\text { and journal } \\
\text { selection }\end{array}$ & Read/review manuscripts of peers & $\begin{array}{l}\text { Peer Feedback: Does the journal } \\
\text { selection fit with the author's intended } \\
\text { message? }\end{array}$ & $\begin{array}{l}\text { Revise manuscript, reformat as } \\
\text { needed for target journal and } \\
\text { category }\end{array}$ \\
\hline $\begin{array}{l}\text { Round } 4 \\
\text { Preparing } \\
\text { for } \\
\text { submission }\end{array}$ & Read/review manuscripts of peers & $\begin{array}{l}\text { Peer Feedback: Final review of paper } \\
\text { before submission } \\
\text { What to expect in the submission } \\
\text { process }\end{array}$ & Submit paper to target journal \\
\hline
\end{tabular}

Table 2: Physician Author Table Characteristics

\begin{tabular}{|c|c|c|}
\hline \multicolumn{2}{|c|}{ Spring $2019(n=5)$} & \multirow{2}{*}{$\frac{n(\%)}{1(20)}$} \\
\hline Condor & Female & \\
\hline Gender & Male & $4(80)$ \\
\hline \multirow{2}{*}{ Position } & Junior faculty & $4(80)$ \\
\hline & Resident & $1(20)$ \\
\hline \multirow{3}{*}{ Location } & California & $2(40)$ \\
\hline & Missouri & $1(20)$ \\
\hline & Ohio & $1(20)$ \\
\hline \multicolumn{3}{|c|}{ Fall $2019(n=4)$} \\
\hline Gender & Female & $2(50)$ \\
\hline Position & Resident & $1(25)$ \\
\hline \multirow{3}{*}{ Location } & California & $2(50)$ \\
\hline & Florida & $1(25)$ \\
\hline & Washington & $1(25)$ \\
\hline
\end{tabular}


Table 3: Writing Rounds Process and Outcomes Measures*

\begin{tabular}{|l|c|c|c|c|c|c|c|}
\hline \multirow{2}{*}{ Number Enrolled } & \multicolumn{5}{|c|}{ Number Attended } & \multirow{2}{*}{$\begin{array}{c}\text { Number of } \\
\text { Submissions }\end{array}$} & $\begin{array}{c}\text { Number of } \\
\text { Publications }\end{array}$ \\
\cline { 3 - 7 } & & Round 1 & Round 2 & Round 3 & Round 4 & & \\
\hline Spring 2019 & 5 & $5(100 \%)$ & $5(100 \%)$ & $5(100 \%)$ & $4(80 \%)$ & $5(100 \%)$ & $1(20 \%)$ \\
\hline Fall 2019 & 4 & $4(100 \%)$ & $4(100 \%)$ & $4(100 \%)$ & $3(75 \%)$ & $2(50 \%)$ & $1(25 \%)$ \\
\hline
\end{tabular}

* Data as of August 17, 2021; we continue to support and track author submissions from each cohort and expect these outcome numbers to increase as the submission and publication process proceeds.

\section{Acknowledgments}

The authors thank Lauren Cafferty, Clinical Research Manager for Military Primary Care Research Network, for her participation and support in feasibility testing; and Dean Seehusen for reviewing an earlier draft of this manuscript. The authors also acknowledge all of the participating physician authors who work outside of their "day jobs" to contribute to the evidence base.

Disclaimer: The views expressed within this publication represent those of the authors and do not reflect the official position of the Henry M. Jackson Foundation, US Air Force, Uniformed Services University of the Health Sciences, or the US Government, the Department of Defense at large.

Presentations: This curriculum was presented at the 2020 Society of Teachers of Family Medicine Annual Conference (virtual).

\section{Corresponding Author}

Jeremy T. Jackson, BA

Military Primary Care Research Network 4301 Jones Bridge Road, Bethesda, MD 20814. 301-295-0703.

jtjackson@hjf.org

\section{Author Affiliations}

Kirsten Winnie, MD - Department of Family Medicine, Uniformed Services University of the Health Sciences | and Travis Air Force Base Family Medicine Residency, Travis AFB, CA

Jeremy T. Jackson, BA - Henry M. Jackson Foundation, Bethesda, MD | and Military Primary Care Research Network, Uniformed Services University of the Health Sciences, Department of Family Medicine, Bethesda, MD Christy J.W. Ledford, PhD - Department of Family Medicine, Uniformed Services University of the Health Sciences, Bethesda, MD

\section{References}

1. Song F, Loke Y, Hooper L. Why are medical and health-related studies not being published? A systematic review of reasons given by investigators. PLoS One. 2014;9(10):e110418.

doi:10.1371/journal.pone.0110418

2. Shah J, Shah A, Pietrobon R. Scientific writing of novice researchers: what difficulties and encouragements do they encounter? Acad Med. 2009;84(4):511-516.

doi:10.1097/ACM.0b013e31819a8c3c

3. Pololi L, Knight S, Dunn K. Facilitating scholarly writing in academic medicine. J Gen Intern Med. 2004;19(1):64-68. doi:10.1111/j.1525-1497.2004.21143.x

4. Rivera JA, Levine RB, Wright SM. Completing a scholarly project during residency training. Perspectives of residents who have been successful. J Gen Intern Med. 2005;20(4):366-369. 
doi:10.1111/j.1525-1497.2005.04157.x

5. Grzybowski SC, Bates J, Calam B, et al. A physician peer support writing group. Fam Med. 2003;35(3):195-201.

6. Chai PR, Carreiro S, Carey JL, Boyle KL, Chapman BP, Boyer EW. Faculty member writing groups support productivity. Clin Teach. 2019;16(6):565-569. doi:10.1111/tct.12923

7. Chan TM, Gottlieb M, Sherbino J, et al. The ALiEM Faculty Incubator: a novel online approach to faculty development in education scholarship. Acad Med. 2018;93(10):1497-1502. doi:10.1097/ACM.0000000000002309

8. Curriculum development for medical education. 2nd ed. Baltimore, MD: Johns Hopkins University Press; 2009.

9. Deci EL, Ryan RM. Self-determination theory: a macrotheory of human motivation, development, and health. Canadian psychology/Psychologie canadienne. 2008;49(3):182 143380431X. doi:10.1037/a0012801

10. Chopra V, Arora VM, Saint S. Will you be my mentor?-Four archetypes to help mentees succeed in academic medicine. JAMA Intern Med. 2018;178(2):175-176. doi:10.1001/jamainternmed.2017.6537

11. Hsieh HF, Shannon SE. Three approaches to qualitative content analysis. Qual Health Res. 2005;15(9):1277-1288. doi:10.1177/1049732305276687

12. Woolliscroft JO. Innovation in Response to the COVID-19 Pandemic Crisis. Acad Med. 2020;95(8):1140-1142. doi:10.1097/ACM.0000000000003402

Copyright $(2021$ by the Society of Teachers of Family Medicine 